\title{
NOUVELLE
}

\section{Organisation dendritique et caractéristiques fonctionnelles des afférences visuelles sur les neurones corticaux}

Nathalie Rochefort

> Les neurones du cerveau communiquent par l'intermédiaire de synapses qui transmettent le signal nerveux d'un neurone à un autre. Un neurone est composé d'un corps cellulaire, d'un axone et de multiples dendrites. II reçoit des signaux (ou afférences) de milliers de synapses qui, ensemble, lui indiquent s'il doit générer une réponse sous la forme d'une décharge de potentiels d'action. Les caractéristiques de cette activité synaptique que les neurones corticaux reçoivent et intègrent pour générer leur réponse demeurent méconnues. Une nouvelle technique d'imagerie permet de visualiser et de cartographier les afférences synaptiques au niveau des dendrites des neurones corticaux dans le cerveau intact de l'animal, in vivo [1]. Cette approche donne des indications sur la façon dont le neurone intègre cette information et génère une réponse spécifique qu'il transmet ensuite aux autres neurones.

Activation préférentielle des neurones du cortex visuel par une orientation spécifique du stimulus visuel

Dans les régions corticales sensorielles, les neurones sont activés de façon préférentielle par un type spécifique de stimulus. Dans le cortex visuel primaire, par exemple, la majeure partie des neurones sont activés de façon sélective en réponse à une orientation spécifique du stimulus visuel, avec une décharge maximale pour une orientation préférentielle.
Ces neurones sont alors appelés «neurones sélectifs à l'orientation ». Certains neurones répondent par exemple fortement à la présentation d'un stimulus visuel ayant une orientation verticale (une barre verticale en mouvement, par exemple) et ne répondent pas du tout à une barre horizontale. D'autres neurones répondent à des contours horizontaux, d'autres encore à des orientations obliques, etc. Depuis la découverte de cette sélectivité par les prix Nobel D. Hubel et T. Wiesel dans les années 1960 [2, 3], les neurones du cortex visuel sont devenus un modèle de choix pour l'étude de l'intégration synaptique. Une question essentielle, et toujours sans réponse, de la physiologie des systèmes sensoriels est: comment les neurones du cortex visuel acquièrent-ils leur sélectivité à l'orientation? En effet, si ces neurones (Figure $1 A$ ) répondent de façon spécifique à une orientation, qu'en est-il de l'information qu'ils reçoivent? Différents scénarios sont envisageables (Figure $1 B-\varepsilon$ ). Les afférences reçues par un neurone (par l'intermédiaire de synapses) pourraient transmettre une information non sélective ou très faiblement sélective à l'orientation (Figure IB) et ce faible biais serait suffisant pour rendre le neurone sélectif à l'orientation. À l'inverse, les afférences pourraient être fortement sélectives à l'orientation. Dans ce cas, diverses organisations spatiales sont possibles. Les afférences avec des préférences d'orientation similaires pour- raient, par exemple, être localisées sur la même dendrite (Figure 1C). En effet, ce type de ségrégation physique serait utile dans le cas où l'activité des différentes entrées synaptiques serait dans un premier temps intégrée localement au niveau des dendrites individuelles, ces dernières constituant alors l'unité computationnelle du neurone. Chaque dendrite enverrait ensuite le résultat de cette intégration au corps cellulaire [4]. Enfin, une autre possibilité serait que les afférences de préférences d'orientation différentes soient dispersées dans tout l'arbre dendritique, sans organisation spatiale évidente (Figure lE). Dans ce cas, l'unité computationnelle de ces neurones serait donc l'arbre dendritique tout entier plutôt que les dendrites individuelles, l'intégration de l'information se faisant au niveau du corps cellulaire. À chaque scénario d'organisation spatiale des afférences synaptiques correspond donc un mode d'intégration de l'information visuelle à des échelles différentes: groupe de synapses, dendrite ou corps cellulaire [4].

Afin de répondre à cette question, il est nécessaire d'enregistrer l'activité des entrées synaptiques (afférences) que reçoit un neurone ainsi que la réponse spécifique de ce neurone. Pour cela, ces enregistrements doivent être effectués in vivo, c'est-à-dire chez l'animal intact auquel on présente un stimulus visuel. Or, au cours des vingt dernières années, les activités synaptiques dans les circuits 


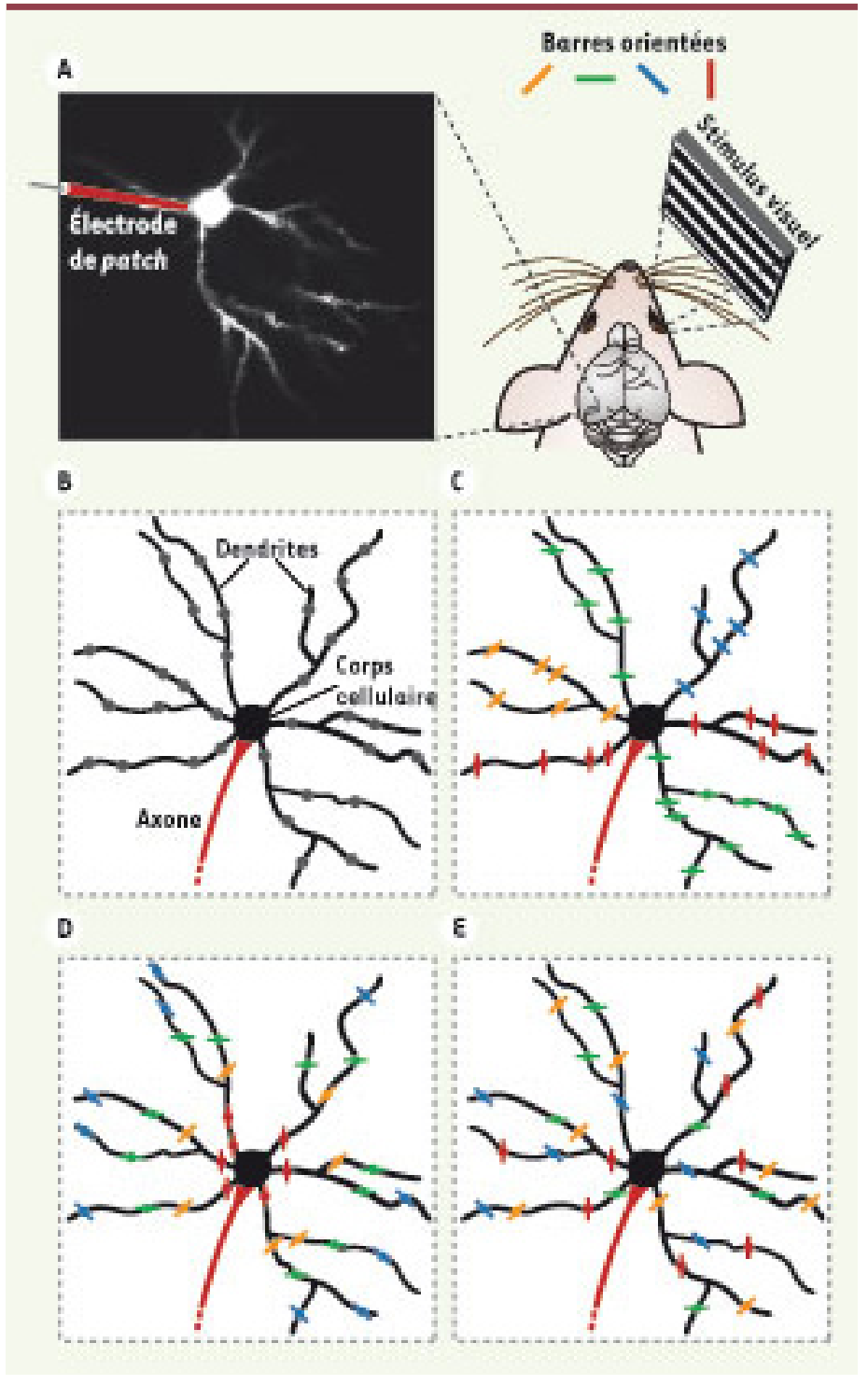

Figure 1. Visualisation et cartographie des afférences visuelles sur un neurone cortical in vivo. A. Dispositif expérimental. La souris anesthésiée est placée devant un écran sur lequel les stimulus visuels (réseaux de barres orientées en mouvement) sont projetés. Une électrode en verre est insérée dans les couches superficielles du cortex visuel primaire et permet d'introduire un marqueur calcique dans un neurone donné. Le marqueur diffuse dans l'ensemble de l'arbre dendritique. Le neurone est hyperpolarisé pour éviter l'apparition de potentiels d'action. Dans ces conditions, un changement de fluorescence localisé au niveau d'une portion de dendrite correspond à l'activité induite par des afférences synaptiques. Panneau de gauche: image d'un neurone marqué in vivo à une profondeur de $150 \mu \mathrm{m}$. Le corps cellulaire et des portions de dendrites sont visibles dans ce plan focal. $B-\varepsilon$. Différents modèles possibles d'organisation dendritique des afférences visuelles. $\boldsymbol{B}$. Les afférences synaptiques ne sont que faiblement sélectives à l'orientation (faible biais en faveur d'une orientation spécifique). C. Les afférences synaptiques ont une forte sélectivité à l'orientation et sont regroupées le long des dendrites en fonction de leur orientation préférentielle. Chaque dendrite reçoit alors des afférences synaptiques d'une orientation spécifique. $\boldsymbol{D}$. Les afférences synaptiques ont une forte sélectivité à l'orientation et sont réparties en fonction de leur orientation préférentielle à une distance donnée du corps cellulaire. L'orientation préférentielle du neurone pourrait correspondre à celle des afférences les plus proches du corps cellulaire. $\varepsilon$. Les résultats expérimentaux montrent que les afférences synaptiques sont fortement sélectives à l'orientation et qu'elles ne sont pas réparties de façon spécifique en fonction de leur orientation préférentielle. Un neurone sélectif à l'orientation peut donc générer une réponse sélective en intégrant l'activité d'afférences synaptiques codant différentes orientations du stimulus visuel et distribuées dans l'ensemble de l'arbre dendritique.

neuronaux ont principalement été étudiées in vitro sur des tranches de cerveau. Ces expériences sur tranches ont révélé des informations essentielles sur la diversité fonctionnelle des synapses, l'intégration synaptique dans les dendrites, les changements d'efficacité synaptique et la contribution de ces différents mécanismes à la formation de la mémoire et au développement du système nerveux. Cependant, ce modèle expérimental a ses limites: de nombreuses connexions neuronales sont sectionnées lors de la préparation de la tranche et il n'est pas possible de tester l'impact de stimulus sensoriels sur l'activité de ces neurones. C'est pourquoi différentes techniques ont été développées pour étudier l'activité des neurones in vivo.

\section{Enregistrements in vivo : \\ électrophysiologie et imagerie biphotonique}

Les enregistrements électrophysiologiques intracellulaires dans le cerveau intact ont apporté des informations précieuses sur l'activité électrique au niveau du corps cellulaire d'un neurone en réponse à un stimulus visuel $[5,6]$. Cependant, ces enregistrements électrophysiologiques ne permettent pas de visualiser l'activité induite par les contacts synaptiques au niveau des dendrites. Seule l'activité globale induite par l'ensemble des afférences est enregistrée au niveau du corps cellulaire. Grâce à l'utilisation de nouvelles techniques d'imagerie, il est désormais possible de visualiser l'activité induite par des afférences synaptiques au niveau des dendrites d'un neurone. Pour cela, une électrode en verre est utilisée pour introduire dans un neurone un marqueur sensible au calcium $[1,7]$. En effet, les flux calciques étant étroitement liés à l'activité électrique neuronale, les enregistrements des variations du calcium libre intracellulaire sont utilisés pour déterminer indirectement l'activité électrique de neurones individuels. Le marqueur calcique introduit dans 
le neurone diffuse dans l'ensemble de l'arbre dendritique et a la propriété de changer de fluorescence en fonction de la concentration intracellulaire de calcium. La technique d'imagerie biphotonique permet de visualiser ces changements de fluorescence in vivo avec une résolution spatiale de l'ordre du micron $[8,9]$. Le principal avantage de cette technique est qu'elle permet d'obtenir des images non seulement à la surface d'un échantillon mais aussi à plusieurs centaines de microns de profondeur. Elle est donc adaptée pour l'imagerie fonctionnelle à haute résolution spatiale dans le cerveau de l'animal, in vivo.

Nous avons utilisé cette approche pour enregistrer les signaux calciques induits par de simples stimulus visuels d'orientations différentes (barres orientées en mouvement) (Figure IA). Le marqueur calcique a été introduit dans des neurones localisés dans les couches superficielles du cortex visuel primaire de souris. En l'absence de potentiels d'action au niveau du corps cellulaire, des signaux calciques locaux ont été enregistrés au niveau de portions $(3,7 \mu \mathrm{m}$ de longueur, en moyenne) de dendrites du neurone étudié, lors de la présentation de stimulus visuels [1]. Ces signaux locaux, appelés hot spots, correspondent à une activité induite par des afférences synaptiques et dépendent de l'activation des récepteurs au glutamate NMDA ( $N$-methyl-D-aspartic acid) [1]. Ainsi, la libération de glutamate au niveau d'afférences synaptiques induit une entrée de calcium par la voie de canaux calciques au niveau de la portion de dendrite étudiée. Cette entrée de calcium est détectée par un changement de fluorescence du marqueur calcique dans la portion de dendrite activée. Grâce à cette approche, il a été possible de déterminer la sélectivité à l'orientation de ces afférences synaptiques et d'observer directement si les afférences synaptiques étaient regroupées anatomiquement selon leur préférence d'orientation.
Hétérogénéité des afférences synaptiques visuelles réparties dans l'ensemble de l'arbre dendritique La majorité des afférences synaptiques sont apparues comme étant sélectives à l'orientation du stimulus. Autrement dit, au niveau d'une dendrite donnée, les signaux calciques locaux (hot spots) ne sont apparus, dans la majorité des cas, que lors de la présentation d'une orientation spécifique du stimulus. Ainsi, un signal calcique local a été enregistré lors de la présentation d'un stimulus d'orientation spécifique (par exemple, verticale) tandis qu'aucun signal n'est apparu lors de la présentation du même stimulus orienté différemment. De plus, cette étude a montré qu'un neurone du cortex visuel reçoit, au niveau de ses dendrites, des afférences synaptiques de préférences d'orientation différentes (Figure $1 \varepsilon$ ), et ce quel que soit son degré de sélectivité à l'orientation. Un neurone est donc capable d'intégrer l'activité d'afférences synaptiques codant différentes orientations et de générer une réponse sélective pour une seule orientation. Cette approche a également montré que les afférences synaptiques partageant la même sélectivité d'orientation (par exemple verticale) étaient distribuées de façon apparemment aléatoire dans l'arbre dendritique des neurones. Aucun regroupement spatial spécifique, par exemple sur une ou plusieurs dendrites, n'a été observé. Les afférences synaptiques de sélectivité d'orientation différente étaient également parsemées, de sorte que des segments dendritiques adjacents pouvaient être activés préférentiellement par des afférences de sélectivité d'orientation différente. Ainsi, les neurones sélectifs à l'orientation peuvent générer une réponse sélective en intégrant l'activité d'afférences synaptiques codant différentes orientations du stimulus visuel et distribuées dans l'ensemble de l'arbre dendritique (Figure $1 \varepsilon$ ).

Par quel mécanisme ces neurones sélectifs à l'orientation intègrent-ils l'activité de l'ensemble des afférences synapti- ques? Une hypothèse est que ces afférences diverses s'additionnent de façon linéaire pour créer, au niveau du corps cellulaire, une activité afférente globale qui ne présente qu'une faible préférence d'orientation. Ce faible biais serait ensuite filtré : seules les réponses les plus importantes, celles évoquées par des stimulus visuels ayant l'orientation préférée, porteraient le potentiel de membrane au-delà du seuil et provoqueraient la décharge de potentiels d'action du neurone. Les résultats décrits ci-dessus indiquent que l'activité afférente globale au niveau du corps cellulaire, qui est peu sélective à l'orientation, ne provient pas d'afférences synaptiques individuelles également non sélectives (Figure IB) mais d'afférences fortement sélectives à l'orientation et de préférences d'orientation différentes (Figure $1 \varepsilon$ ). Différents mécanismes d'intégration de cette information hétérogène peuvent être envisagés et seront certainement l'objet de recherches futures.

Ces résultats donnent également des indices sur les règles qui régissent le développement des connexions neuronales dans le cortex visuel. Dans le cortex visuel des rongeurs, les neurones ayant des sélectivités d'orientation différentes sont distribués de façon aléatoire [10] et non pas organisés en colonnes comme chez les mammifères supérieurs (chat, furet, primates, par exemple) $[3,11$, 12]. L'arrangement apparemment aléatoire des afférences synaptiques décrit ci-dessus pourrait donc provenir du fait que chaque neurone reçoit des afférences synaptiques de ses voisins de façon aléatoire. Dans ce cas, les connexions sur un neurone ne se feraient pas par un processus de sélection des afférences visuelles en fonction de la préférence d'orientation finale de ce neurone.

\section{Perspectives}

Quelles sont les règles régissant la connectivité neuronale, comment les neurones acquièrent-ils leur sélectivité à certaines caractéristiques des stimulus? La méthode décrite ci-dessus 
permettra sans doute d'apporter quelques réponses grâce à l'analyse détaillée des afférences sensorielles reçues par différents types de neurones. En effet, il est possible que d'autres types neuronaux, en particulier ceux présentant une dendrite apicale proéminente [13], ou ceux d'autres espèces (organisés en colonnes d'orientation), présentent une organisation spatiale différente de leurs afférences synaptiques, avec par exemple la présence de clusters capables de générer des potentiels d'action dendritiques [14]. Pour l'instant, seule l'activité d'une portion restreinte de l'arbre dendritique a pu être enregistrée (environ $10 \%$ ). Mais les progrès constants en imagerie biphotonique permettent d'envisager que, dans un futur proche, il sera possible d'enregistrer l'activité de l'ensemble des afférences synaptiques que reçoit un neurone et de mieux com- prendre les mécanismes d'intégration de l'information sensorielle [15]. $\diamond$

Dendritic organization and functional characteristics of visually evoked inputs to cortical neurons in vivo

\section{CONFLIT D'INTÉRÊTS}

Les auteurs déclarent n'avoir aucun conflit d'intérêts concernant les données publiées dans cet article.

\section{RéFÉRENCES}

1. Jia H, Rochefort NL, Chen X, Konnerth A. Dendritic organization of sensory input to cortical neurons in vivo. Nature 2010 ; 464 : 1307-12.

2. Hubel DH, Wiesel TN. Receptive fields of single neurones in the cat's striate cortex.J Physiol 1959; 148: 574-91.

3. Hubel DH, Wiesel TN. Receptive fields, binocular interaction and functional architecture in the cat's visual cortex.J Physiol 1962 ; 160 : 106-54.

4. Häusser M, Mel B. Dendrites: bug or feature? Curr Opin Neurobiol 2003; 13 : 372-83.

5. Bringuier V, Chavane F, Glaeser L, Fregnac Y. Horizontal propagation of visual activity in the synaptic integration field of area 17 neurons. Science 1999 ; 283: 695-9.
6. Carandini M, Ferster D. Membrane potential and firing rate in cat primary visual cortex. J Neurosci 2000 . $20: 470-84$.

7. Kitamura K, Judkewitz B, Kano M, et al. Targeted patch-clamp recordings and single-cell electroporation of unlabeled neurons in vivo. Nat Methods 2008; 5 : 61-7.

8. Denk W, Strickler JH, Webb WW. Two-photon laser scanning fluorescence microscopy. Science 1990 ; $248: 73-6$.

9. Helmchen F, Denk W. Deep tissue two-photon microscopy. Nat Methods $2005 ; 2$ : 932-40.

10. Ohki K, Chung S, Ch'ng YH, et al. Functional imaging with cellular resolution reveals precise microarchitecture in visual cortex. Nature 2005 ; 433 : 597-603.

11. Hubel DH, Wiesel TN. Receptive fields and functional architecture of monkey striate cortex.J Physiol 1968 ; $195: 215-43$.

12. White $L \varepsilon$, Fitzpatrick D. Vision and cortical map development. Neuron 2007; $56: 327-38$.

13. Larkum ME, Nevian T, Sandler M. Synaptic integration in tuft dendrites of layer 5 pyramidal neurons: a new unifying principle. Science 2009 ; $325:$ 756-60.

14. Larkum ME, Nevian T. Synaptic clustering by dendritic signalling mechanisms. Curr Opin Neurobiol 2008 ; 18: 321-31.

15. Férézou I. Combien de neurones pour une sensation? Med Sci (Paris) $2008 ; 24: 802-4$.

\section{NOUVELL}

\section{Enfin un modèle de culture du virus de l'hépatite C adapté aux hépatocytes humains primaires!}

Arielle R. Rosenberg,

Arnaud Carpentier, Philippe Podevin

Université Paris Descartes,

EA 4474 Virologie de l'hépatite C,

22, rue Méchain, 75014 Paris, France. arielle.rosenberg@parisdescartes.fr
Le virus de l'hépatite $C$, un paradigme des virus difficiles à cultiver

Grâce à des méthodes prenant le contrepied de la virologie classique, le virus de l'hépatite C (VHC) fut le premier virus de I'histoire de la virologie à avoir été identifié d'abord par clonage de son génome avant d'avoir été observé - «entrevu», devrions-nous dire - en microscopie électronique et bien avant d'avoir pu être cultivé [1]. Témoin de la puissance de la biologie moléculaire, cette découverte de la toute fin des années 1980 permit rapidement la mise au point du premier test sérologique de dépistage, freinant ainsi l'incidence de l'infection. Mais la biologie moléculaire a aussi ses limites. Comme tout virus, le VHC est un parasite intracellulaire obligatoire, détournant le métabolisme de la cellule qu'il infecte au profit de sa propre multiplication. Pour identifier les acteurs cellulaires usurpés par le virus et étudier les conséquences de l'infection sur la physiologie cellulaire, la priorité du virologiste est donc de chercher à cultiver le virus, c'est-à-dire de reproduire l'ensemble des étapes du cycle viral, y compris la production de nouvelles particules infectieuses, dans des cellules in vitro.
Le VHC étant un virus humain hépatotrope, il paraissait à la fois logique et souhaitable de recourir à des cultures primaires d'hépatocytes humains adultes, qui représentent le modèle in vitro le plus proche de la cellule hôte du virus au cours de l'infection naturelle. Isolés habituellement à partir des reliquats macroscopiquement sains de pièces d'hépatectomie partielle, les hépatocytes humains peuvent en effet être maintenus in vitro pendant quelques jours ou semaines dans un état quiescent et fortement différencié. Dès la fin des années 1990, plusieurs équipes ont inoculé de telles 